\title{
(W-R)-MATROIDS AND THIN SCHUBERT-TYPE CELLS ATTACHED TO ALGEBRAIC TORUS ACTIONS
}

\author{
YI HU
}

(Communicated by Jeffry N. Kahn)

\begin{abstract}
Given a projective variety acted on by an algebraic torus, we introduce the notion of $(\mathbf{W}, \mathbf{R})$-matroids using the fixed-point set $\mathbf{W}$ and the set $\mathbf{R}$ of equivalence classes of one-parameter subgroups. The (W, $\mathbf{R})$-matroids provide close links among the geometry of torus orbits and Schubert-type cells, the theory of momentum polyhedra, and the combinatorial geometries. On the way to establishing the main theme of the paper, we showed that there are only finitely many Bialynicki-Birula decompositions induced by infinitely many one-parameter subgroups.
\end{abstract}

\section{INTRODUCTION}

0.1 . In this paper we consider certain combinatorial objects attached to a projective algebraic variety $X$ with an action of an algebraic torus $T=\left(\mathbb{C}^{*}\right)^{n}$ and the decompositions of $X$ into orbits with the same geometry. Our results specialize to some results of [GGMS] and [GS] for compact homogeneous space $G / P$ and also specialize to some of [KSZ1] for Chow varieties.

0.2. Gelfand-Goresky-MacPherson-Serganova [GGMS] in the case of Grassmann manifold and Gelfand-Serganova [GS] in the case of a general homogeneous space $G / P$ have proved that the following two decompositions of $G / P$ into strata coincide:

(1) The union of all the orbits of the Cartan subgroup whose images under the momentum map are the same convex polyhedron.

(2) The intersection of the Schubert cells for all Borel subgroups that contain the given Cartan subgroup.

0.3. For simplicity, let the projective variety $X$ be smooth for a moment. Assume we are given an algebraic torus $T$ acting on $X$ with isolated fixed points. Let $\mathbf{W}$ denote the fixed-point set. Take a generic one-parameter subgroup $a$ of $T$ with parameter $\lambda$ so that it has the same fixed-point set $W$ as that of the whole group $T$. For each $w \in \mathbf{W}$, one defines, following [B-B], its contracting

Received by the editors January 6, 1993 and, in revised form, June 18, 1993.

1991 Mathematics Subject Classification. Primary 14L30; Secondary 52B40, 05 B35. 
set:

$$
X_{w}=\left\{x \in X \mid \lim _{\lambda \rightarrow 0} \lambda \cdot x=w\right\}
$$

Then it is known that $X_{w}$ form a cell decomposition $X=\bigcup_{w \in \mathbf{W}} X_{w}$ where each $X_{w}$ is $a$-equivariantly isomorphic to an affine space. The above decomposition specializes to the famous Bruhat decomposition. In other words, when $X$ is the flag variety $G / P$ and $a$ is a generic 1-dimensional subgroup of a Cartan subgroup, then each $X_{w}$ is just a Schubert cell. For this reason, $X_{w}$ is often referred to as a Schubert-type cell for the action of $a$ and $\overline{X_{w}}$ is henceforth referred to as a Schubert-type variety.

We are able to prove a similar result for the projective variety $X$. That is, the following two decompositions of $X$ into strata coincide:

(1) The union of all the orbits of the torus $T$ whose images under a momentum map are the same convex polyhedron.

(2) The intersection of the Schubert-type cells which are obtained by permuting the generic one parameter subgroups.

0.4. ( $W, Q$ )-matroids, introduced by Gelfand and Serganova in [GS], are certain combinatorial objects attached to a finite Coxeter group $W$ and a parabolic subgroup $Q$. By definition, a $(W, Q)$-matroid is a subset of $W / Q$ which contains a unique minimal (or, equivalently, a unique maximal) element under every Bruhat partial order. For homogeneous space $G / P$, the $(W, Q)$-matroids describe the partition of $G / P$ into the union of thin Schubert cells. Note that $W / Q$ can be identified with the fixed-point set of the Cartan subgroup.

We are able to generalize the notion of $(W, Q)$-matroids to an algebraic torus action. To achieve this, we define a parametrizing set $\mathbf{R}$ and for each element of $\mathbf{R}$ a partial order on the fixed-point set $\mathbf{W}$ in analogy of a Bruhat order. Then we define a $(\mathbf{W}, \mathbf{R})$-matroid to be any subset of $\mathbf{W}$ that contains a unique minimal (or, equivalently, a unique maximal) element under every analogous Bruhat partial order. It will be clear that the notion of $(\mathbf{W}, \mathbf{R})$-matroids specializes to that of $(W, Q)$-matroids of [GS] and that of hypermatroid of [KSZ1]. Also (W, R)-matroids are closely related to "thin cells" (see Corollary 6.4 and Remark 5.5).

\section{Preliminaries on homogeneous space $G / P$}

1.1. The notion of torus strata was first introduced for the actions of the maximal tori on Grassmannians in [GGMS]. The importance of this notion lies in the fact that it establishes remarkable connections among the geometry of Schubert cells in the Grassmann manifold, the theory of convex polyhedra, and the theory of matroids (or combinatorial geometries in the sense of Crapo and Rota [CR]). In [GGMS], the authors proved that the following three different decompositions of the Grassmannian into strata (torus strata or matroid strata as in [S]) all coincide:

(1) The set of points in the Grassmannian such that the corresponding projective configuration represents a fixed combinatorial geometry (matroid).

(2) The union of the orbits of the maximal torus whose projection under a momentum map is a fixed convex polyhedron. 
(3) A multi-intersection of translates of Schubert cells which are obtained by permuting the coordinate axes.

1.2. In [GS], Gelfand and Serganova further explored the combinatorics of the maximal torus action on a homogeneous space $G / P$. In this category, the last two characterizations of a torus stratum remain equivalent. However, the first characterization of a torus stratum is dropped out because an ordinary matroid no longer fits into the theory. Instead, they defined, for an arbitrary homogeneous space, something weaker than a matroid, namely, the so-called $(W, Q)$-matroid. This $(W, Q)$-matroid no longer characterizes a torus stratum. However, the orbits on a fixed stratum still represent the same $(W, Q)$-matroid.

\subsection{See $\S 7$ for hypermatroids of [KSZ1].}

\section{Partition by torus strata}

2.1. Our primary goal in this paper is to generalize some of the results in [GGMS] and [GS]. We consider any projective variety $X$ with an algebraic action of a complex torus $T$. Note that the characterization 1.1.(2) can still be used to establish the notion of torus stratum on a projective variety with an action of an algebraic torus.

2.2. Let $X$ be a projective algebraic variety (may be singular) with an effective action of an algebraic torus $T=\left(\mathbb{C}^{*}\right)^{n}$. Assume the torus action extends to a linear action on the ambient projective space $\mathbb{P}^{N}$. Choose a Kähler metric on $\mathbb{P}^{N}$ which is invariant under the compact torus $T_{c}=\left(S^{1}\right)^{n} \subset\left(\mathbb{C}^{*}\right)^{n}$. Let

$$
\mu: X \rightarrow \mathbb{R}^{n}
$$

be an associated moment map. Then it is known that $\mu(\overline{T \cdot x})$ for any $x \in X$ is a convex polyhedron in $\mathbb{R}^{n}$ whose vertices are the moment map images of the fixed points contained in $\overline{T \cdot x}$ ([A], [GuS]). For simplicity, we shall assume that the torus action considered in this paper has finitely many fixed points, although the results can easily be generalized to the general case (one can use finite connected components to take the role of finite fixed points).

There is a decomposition of $X$ into invariant subspaces, $X=\bigcup_{C \in \Xi} X^{C}$, which we describe as follows:

a point $x$ is in the stratum $X^{C}$ if and only if $\mu(\overline{T \cdot x})=C$.

Note that $\Xi$ is a collection of polyhedra in $\mathbb{R}^{n}$. So two orbits are in the same stratum if and only if their closures contain the same fixed points.

2.3. Definition. Each above stratum $X^{C}$ is called a torus stratum. The decomposition of $X$ into the union of torus strata is called the torus stratification of $X$.

2.4. Example. When $X$ is a toric variety containing $T$ as a dense orbit, then the torus stratification is simply the decomposition by $T$-orbits.

2.5. Example. When $X$ is the Grassmannian of $k$-subspaces in an ambient $n$-complex space and the complex torus $\left(\mathbb{C}^{*}\right)^{n}$ acts on it by multiplying coordinates, then the torus strata are in one-to-one correspondence with the rank $k$ C-representable matroids [GGMS]. The realizability problem of matroids is 
very difficult. One may consult [S] for more interesting results on matroids and Grassmannians, among other things.

\section{The SET $\mathbf{R}$ AND ITS ASSOCIATEd FAN}

3.1. Definition. A one-parameter subgroup of $T$ is said to be generic if it has the same fixed points as that of $T$.

Now we assume that $X$ is smooth (see $\S 7$, however). Given a generic 1parameter subgroup $a$, the Bialynicki-Birula cellular decomposition theorem asserts that such a 1-parameter subgroup gives rise to a cell-decomposition of $X$ where each cell contains a unique fixed point (see 0.3 ). Let $\mathbf{W}$ be the fixed-point set which is finite by the assumption. Let $X=\bigcup_{w \in \mathbf{W}} X_{w}^{a}$ be the corresponding cellular decomposition where $X_{w}^{a}$ is the cell that contains $w \in \mathbf{W}$. Among interesting examples of such spaces are $G / P$, toric varieties ([D]), complete symmetric varieties of De Concini and Procesi, and Chow varieties and Hilbert schemes of [KSZ1] (see $\S 7)$.

3.2. Definition. Two one-dimensional subgroups are said to be equivalent if they give the same cellular decompositions. We use $\mathbf{R}$ to denote the set of equivalent classes of generic one-parameter subgroups of $T$.

The above equivalence relation defines a fan of cone decomposition of $\operatorname{Hom}\left(\mathbb{C}^{*}, T\right) \otimes \mathbb{R} \cong \mathbb{R}^{n}$. That is, two elements of $\operatorname{Hom}\left(\mathbb{C}^{*}, T\right)$ are equivalent if and only if they are in a same cone. And the set $\mathbf{R}$ can be identified with the set of maximal cones in this fan. Inspired by an e-mail correspondence with Borovik, we would like to study this fan. The author should like to thank the referee for requesting a complete description of this fan. Denote this fan by $\Sigma_{\mathbf{R}}$. Let $\Sigma_{\mu}$ be the dual fan of the rational polytope $\mu(X)$. (For simplicity, we shall identify $\operatorname{Hom}\left(\mathbb{C}^{*}, T\right) \otimes \mathbb{R}$ and $\operatorname{Hom}\left(T, \mathbb{C}^{*}\right) \otimes \mathbb{R}$ with $\mathbb{R}^{n}$.) In general, $\Sigma_{\mathbf{R}}$ is finer than the dual fan $\Sigma_{\mu}$ of the polytope $\mu(X)$. We need a few terminologies to proceed.

3.3. An arrangement of hyperplanes in $\mathbb{R}^{n}$ is a collection of finite hyperplanes of $\mathbb{R}^{n}$. It is called central if their intersection is nonempty and consists of a point. This point is referred to as the center of the arrangement. Without loss of generality, this center can be assumed to be the origin (after a translation if necessary).

3.4. Definition. A fan $\Sigma$ is of arrangement-type if whenever $\mathbb{R}_{\geq 0} v\left(v \in \mathbb{R}^{n}\right)$ is an edge, so is $\mathbb{R}_{\leq 0} v$. In this case, the set of edges induces a central arrangement of hyperplanes.

A remarkable property of such fans is that they form a tail of all fans with respect to the partial order by subdivision. That is, any fan has a subdivision that is of arrangement-type. To see this, we simply add an edge $\mathbb{R}_{\leq 0} v=\mathbb{R}_{\geq 0}(-v)$ for each edge $\mathbb{R}_{>0} v$ of the given fan $\Sigma$. Clearly we obtain a subdivision this way. We call this subdivision the arrangement-type extension of $\Sigma$. This is the minimal subdivision of $\Sigma$ that is of arrangement-type. A fan of arrangementtype admits an involution $\theta=-1$. Such a fan may therefore be characterized as it is a fan which is invariant under $\theta=-1$. Note that the fan formed by Weyl chambers of a Coxeter group is of arrangement-type. 
Every central arrangement of hyperplanes induces a fan. It may be an interesting combinatorial problem to consider relations between suitable central arrangements of subspaces (of possibly various dimensions) and their corresponding fans.

The following theorem simply asserts that the fan $\Sigma_{\mathbf{R}}$ is the coarsest fan of hyperplane arrangement refining the fan $\Sigma_{\mu}$.

3.5. Theorem. The fan $\Sigma_{\mathbf{R}}$ is the arrangement-type extension of the fan $\Sigma_{\mu}$. In particular, $\Sigma_{\mathbf{R}}=\Sigma_{\mu}$ if and only if $\Sigma_{\mu}$ is of arrangement-type.

Proof. Let $\Sigma^{\prime}$ be the arrangement-type extension of $\Sigma_{\mu}$. We only need to show two generic 1-parameter subgroups are equivalent if and only if they lie in the same maximal cone of $\Sigma^{\prime}$. Let $v$ be the vector field on $X$ generating the $S^{1}$ action on $X$ arising from the action of a one-parameter subgroup $a$, by restricting to the unit circle. Let $\omega$ be an $T_{c}$-equivariant Kahler 2-form on $X$, and let $i_{v} \omega$ be the 1 -form obtained by contraction. This 1 -form is exact since $H^{1}(X)=0$. Hence there is a smooth function $f$ on $X$ such that $i_{v} \omega=d f$. The function $f$ is known to be a Morse function whose critical points are exactly the fixed points of the $a$-action. Moreover, the cell decomposition of $X$ associated to $f$ via the Morse theory coincides with the Bialynicki-Birula decomposition $X=\bigcup_{w \in \mathbf{W}} X_{w}^{a}$. In fact, the function $f$ is just the moment map associated to the action $a$, which is the moment map $\mu$ followed by the projection to $\mathbb{R} a$

$$
f: X \stackrel{\mu}{\rightarrow} \mathbb{R}^{n} \stackrel{\pi}{\rightarrow} \mathbb{R} a
$$

where $\pi$ is the orthogonal projection. Now $x$ is in the cell $X_{w}^{a}$ if and only if $x$ flows to $w$ under $-\operatorname{grad} f$ if and only if $f(w)$ is minimal in $f(\overline{a \cdot x})$. The last characterization only depends on the maximal cone in which $a$ lives. That is, if $a$ and $b$ are in the same maximal cone of $\Sigma^{\prime}$ and $f$ and $g$ are their corresponding moment maps (Morse functions), then $g(w)$ is minimal in $g(\overline{a \cdot x})$ whenever $f(w)$ is minimal in $f(\overline{a \cdot x})$ and vice versa. This implies that $a$ and $b$ define the same cell-decomposition. It is clear that points on different cones must define different cell-decompositions. This completes the proof.

3.6. Example. For every $G / P, \Sigma_{\mathbf{R}}$ is the fan formed by Weyl chambers which is in general finer than $\Sigma_{\mu}$. And $\mathbf{R}$ is precisely the set of Weyl chambers. Of course, in a good case such as $G / B, \Sigma_{\mathbf{R}}=\Sigma_{\mu}$ and the set $\mathbf{R}$ may be identified with the fixed-point set $\mathbf{W}$.

\section{INCIDENT RELATION AND PARTIAL ORDERS ON THE FIXED POINT SET}

4.1. In addition to the decomposition $X=\bigcup_{w \in \mathbf{W}} X_{w}^{a}$, which is referred to as the plus-decomposition, there is a minus-decomposition which can be obtained by changing the parameter of $a$ from $\lambda$ to $\lambda^{-1}$. Let the newly introduced one-parameter subgroup be denoted by $\bar{a}$ and its corresponding cellular decomposition be denoted by $X=\bigcup_{w \in \mathbf{W}} X_{w}^{a}$; then

$$
X_{w}^{a}=\left\{x \in X \mid \lim _{\lambda^{-1} \rightarrow 0} \lambda^{-1} \cdot x=w\right\}=\left\{x \in X \mid \lim _{\lambda \rightarrow \infty} \lambda \cdot x=w\right\} .
$$


This minus-decomposition coincides with the cell decomposition associated to the Morse function $(-f)$ via the Morse theory.

4.2. Proposition. The following statements are equivalent:

(1) $X_{u}^{a} \cap \overline{X_{v}^{a}} \neq \emptyset$.

(2) $X_{v}^{\bar{a}} \cap \overline{X_{u}^{a}} \neq \emptyset$.

(3) $X_{v}^{a} \cap X_{u}^{a} \neq \emptyset$.

Proof. We first show that (3) implies (1) and (2). Let $x$ be a point on $X_{v}^{a} \cap X_{u}^{a}$ and $\lambda$ be the parameter of the one-parameter group of $a$. Then by the definition we have

$$
\lim _{\lambda \rightarrow \infty} \lambda \cdot x=u \text { and } \lim _{\lambda \rightarrow 0} \lambda \cdot x=v .
$$

The first identity implies that $u$ is in the closure of $X_{v}^{a}$, hence $X_{u}^{a} \cap \overline{X_{v}^{a}} \neq \emptyset$. Likewise, the second identity implies that $v$ is in the closure of $X_{u}^{a}$, hence $X_{v}^{\bar{a}} \cap \overline{X_{u}^{a}} \neq \emptyset$.

On the other hand, to prove that (1) implies (3), notice that since the fixed point $u$ must be contained in the closure of $X_{v}^{a}$, there must be a point $x \in X_{v}^{a}$ such that the $\mathbb{C}^{*}$ orbit closure $\overline{a \cdot x}$ contains $u$. This implies that $\lim _{\lambda \rightarrow \infty} \lambda \cdot x=u$. That is, $x \in X_{u}^{a}$. Hence, $x \in X_{v}^{a} \cap X_{u}^{a}$. Similarly one can prove that (2) implies (3).

4.3. Definition. The element $a$ induces a partial order $\prec^{a}$ on $\mathbf{W}: u \prec^{a} v$ if and only if one of the three equivalent conditions in Proposition 4.2 is satisfied.

4.4. Example. In the case of flag variety $G / P$, the partial order above is simply a Bruhat order. The parametrizing set $\mathbf{R}$ of partial orders is identical to the corresponding (full) Weyl group. In general, the (analogous) Bruhat order is not a linear order even for simple varieties such as toric varieties. Of course, when $\left(\mathbb{C}^{*}\right)^{n}$ acts on $\mathbb{P}^{n}$ as usual, the Bruhat order is linear.

\section{5. (W, R)-MATROIDS}

5.1. Recall that we use $\mathbf{R}$ to denote the finite set of equivalent classes of generic one-parameter subgroups where two one-parameter subgroups are said to be equivalent if they give the same cellular decomposition of $X$ (3.1 and 3.5). There is a poset structure on the fixed-point set $\mathbf{W}$ attached to every element of $\mathbf{R}$ (see 4.3). Let $L \subset \mathbf{W}$ be the set of the fixed points of an orbit closure; then in the sequel we shall prove that $(\mathbf{W}, \mathbf{R} ; L)$ satisfies the "condition of minimality" and the "condition of maximality".

5.2. Definition. Let $L \subset \mathbf{W}$ be an arbitrary set of $\mathbf{W}$. An element $s \in L$ is called $a$-minimal in $L$ if $s \leq^{a} u$ for all $u \in L$. We shall say that $L$ satisfies the condition of minimality if there is a unique $a$-minimal element in $L$ for every $a \in \mathbf{R}$. Similarly we can define the corresponding notions for maximality.

5.3. Lemma. The condition of minimality is equivalent to the condition of maximality.

Proof. Let $a$ be an element of $\mathbf{R}$ which is a representative of an equivalent class of one-parameter subgroups. Consider the group homomorphism: $\lambda \rightarrow$ $\lambda^{-1}$. Then this homomorphism and $a$ induce another one-parameter subgroup. 
We denote it $\bar{a}$. Then $a$-maximality implies $\bar{a}$-minimality and vice versa by Bialynicki-Birula's (+)-decomposition and (-)-decomposition.

5.4. Definition. A $(\mathbf{W}, \mathbf{R})$-matroid is a triple $(\mathbf{W}, \mathbf{R} ; L)$ where $L$ is a subset of $\mathbf{W}$ such that $L$ satisfies the condition of minimality (or, maximality).

5.5. Remark. Given a $(\mathbf{W}, \mathbf{R})$-matroid $L$, there is an attached function $f$ : $\mathbf{R} \rightarrow \mathbf{W}$ defined as follows: $f(a)$ is the $a$-maximal element in $L$ for each $a \in \mathbf{R}$. Likewise, there is another function $f^{\prime}: \mathbf{R} \rightarrow \mathbf{W}$ which assigns the $a$ minimal element of $L$ to $a$ for every $a \in \mathbf{R}$. Following Borovik-Gelfand, one may call such a map a combinatorially convex map. In the case that $\mathbf{R}=\mathbf{W}$, that is, when $\Sigma_{\mathbf{R}}=\Sigma_{\mu}$ (e.g., flag varieties $G / B$ ), the map $f$ is a self-map that is a retraction with respect to $L$. That is, $f(\mathbf{W})=L \subset \mathbf{W}$ and $\left.f\right|_{L}=$ identity. One can say something similar for the map $f^{\prime}$.

5.6. Definition. For convenience, sometimes we refer the finite set $\mathrm{W} \cap \overline{H \cdot x}$ $(x \in X)$ as the list of $x$ and the list of a torus stratum is defined to be the list of any point in it. So two points are in the same stratum if and only if they have the same list.

5.7. Theorem. Let $L$ be the list of some $x \in X$. Then $(\mathbf{W}, \mathbf{R}, L)$ is a $(\mathbf{W}, \mathbf{R})$ matroid.

Proof. To prove this theorem, notice that Proposition 4.2 leads to the following claim. Let $a$ be an element in $\mathbf{R}, u$ be an element of $\mathbf{W}$, and $X_{u}^{a}$ be the cell determined by $a$ and $u$. Then we have

$$
\mu\left(\overline{X_{u}^{a}}\right)=\text { convex hull of }\left\{\mu(v) \mid v \in \mathbf{W}, v \leq^{a} u\right\} \subset \mathbb{R}^{n} .
$$

Now given $a \in \mathbf{R}$, we have $X=\bigcup_{v \in \mathbf{W}} X_{v}^{a}$. Therefore, $x \in X_{w}^{a}$ for some $w \in \mathbf{W}$. Thus

$$
L=\overline{T \cdot x} \cap \mathbf{W} \subset\left\{u \in \mathbf{W} \mid u \leq^{a} w\right\}
$$

because $\overline{T \cdot x} \subset \overline{X_{w}^{a}}$. Now $w \in \overline{T \cdot x}$ (since $\left.\lim _{\lambda \rightarrow 0} \lambda \cdot x=w\right)$, thus $w$ is the unique maximal element in $L$.

The converse of Theorem 5.7 is not true. That is, some $(\mathbf{W}, \mathbf{R})$-matroids may not be the lists of torus strata [GS].

5.8. Example. A Coxeter group $W$ together with its Bruhat orders is a prototype for our problem. Hence our result generalizes that of Gelfand and Serganova on $(W, Q)$-matroids.

5.9. Example. Let $X$ be a projective toric variety containing $T$ as a dense orbit. Let $S$ be a general subtorus of $T$. Then all $(\mathbf{W}, \mathbf{R})$-matroids $L$ arise from the lists of points in $X$. In particular, the set of $(\mathbf{W}, \mathbf{R})$-matroids is in one-to-one correspondence with the set of $T$-orbits.

\section{Partition by thin Schubert-type cells}

6.1. Lemma. Let $a \in \mathbf{R}$ and $u \in \mathbf{W}$; then the cell $X_{u}^{a}$ is a union of torus strata.

Proof. It suffices to prove that if $X^{D} \cap X_{u}^{a}$ is not empty, then $X^{D}$ is contained in $X_{u}^{a}$. For this purpose, we assume that there exists a point $y$ in $X^{D}$ but 
$y$ is not in $X_{u}^{a}$. Let $x$ be a point in the intersection of $X^{D}$ and $X_{u}^{a}$. Then $\mathbf{W} \cap \overline{T \cdot x}=\mathbf{W} \cap \overline{T \cdot y}$. Now because $y$ is not in $X_{u}^{a}$, there exists an element $w$ in $\mathbf{W}, w$ is different from $u$, such that $y$ is in $X_{w}^{a}$. So $w$ is the unique maximal element in $\mathbf{W} \cap \overline{T \cdot y}$ by the proof of Theorem 5.7. Meanwhile, by the same reason, $u$ is the unique maximal element of $\mathrm{W} \cap \overline{T \cdot x}$. So we conclude that $u=w$ since $\mathbf{W} \cap \overline{T \cdot x}=\mathbf{W} \cap \overline{T \cdot y}$, which is a contradiction.

6.2. Definition. For each element $a \in \mathbf{R}$, we choose precisely one cell associated to $a$. Then we call the intersection of all the chosen cells a thin cell provided that it is not empty.

The following theorem generalizes the corresponding results of [GGMS] and [GS] from homogeneous spaces to a nonsingular projective variety with a torus action.

6.3. Theorem. The partition of $X$ into thin cells coincides with the torus stratification.

Proof. Given a torus stratum $X^{C}$, for any element $a \in \mathbf{R}$, by Theorem 5.7, the list of this torus stratum has a unique $a$-maximal element, denoted by $f(a)$. Thus $X^{C}$ is contained in $X_{f(a)}^{a}$ by the proof of Lemma 6.1. Hence we have that

$$
X^{C} \subset \bigcap_{a \in \mathbf{R}} X_{f(a)}^{a} .
$$

It is clear that any stratum contained in $\bigcap_{a \in \mathbf{R}} X_{f(a)}^{a}$ has the same list $\{f(a) \mid a \in$ $\mathbf{R}\}$, hence by Lemma 6.1 again, $X^{C}=\bigcap_{a \in \mathbf{R}} X_{f(a)}^{a}$. On the other hand, if $\bigcap_{a \in \mathbf{R}} X_{f(a)}^{a}$ is not empty, then it has to be a single stratum by using Lemma 6.1 and the fact that all of its element must have the same list.

6.4. Corollary. Every thin cell (i.e, a torus stratum) is a multi-intersection of Schubert-type cells of the form $\bigcap_{a \in \mathbf{R}} X_{f(a)}^{a}$ where $f: \mathbf{R} \rightarrow \mathbf{W}$ is a convex function which assigns the a-maximal element of $\mathbf{W} \cap \overline{T \cdot x}$ to $a$. We have, in particular, $f(\mathbf{R})=\mathbf{W} \cap \overline{T \cdot x} \subset \mathbf{W}$.

Proof. It is clear from the proof of Theorem 6.3.

\section{ON Singular varieties, ChOW varieties, and Hilbert SCHEmes}

7.1. In this section, we observe that our whole theory can be extended to the singular case without encountering any (genuine) additional difficulty. The author is grateful to the referee, who predicted the above and pointed out that our results should be compared with those of [KSZ1].

Note that $\S 2$ is established for all projective varieties. We can still define the Bialynicki-Birula's contracting set as

$$
X_{w}=\left\{x \in X \mid \lim _{\lambda \rightarrow 0} \lambda \cdot x=w\right\} .
$$

Now $X_{w}$ may have singularities. So it may not be an affine space but is still a contractible set. No proofs or arguments really need smoothness. The proof of Theorem 3.5 is valid without mentioning that the composite $\pi \cdot \mu$ is a Morse 
function. Thus our results in previous sections extend naturally to singular varieties.

7.2. In [KSZ1], the authors consider the Chow variety $G(k, d, n)$ which is defined as the set of all cycles in $\mathbb{P}^{n-1}$ of dimension $k-1$ and degree $d$. These varieties contain the Grassmannian $G\left(k, \mathbb{C}^{n}\right)$ as its special case (the case of $d=1)$. The torus $T=\left(\mathbb{C}^{*}\right)^{n-1}$ acts naturally on the Chow variety via multiplying the coordinates of $\mathbb{P}^{n-1}$. Using the notion of Chow form and weight polytope, the authors defined the Chow polytope $\mathrm{CH}(Z)$ for any cycle $Z$ as the weight polytope of its Chow form. Again we use $\mu$ to denote the moment map associated to the action of $T$ on $G(k, d, n)$. The following is essentially due to [KSZ1].

7.3. Proposition. The polytope $\mu(\overline{T \cdot Z})$ coincides with the Chow polytope $\mathrm{CH}(Z)$.

Proof. It follows from the definitions of moment map and Chow polytope.

In [KSZ1, Remark 2.11], a hypermatroid polytope of rank $k$ and degree $d$ is also defined. The set of the vertices of such a polytope may be defined as a hypermatroid. With this in mind a hypermatroid is thus equivalent to $(\mathbf{W}, \mathbf{R})$ matroid. This property is first observed in [GS] for generalized flag variety $G / P$. From this point of view, our $(\mathbf{W}, \mathbf{R})$-matroid may be considered as an extention of hypermatroid polytope from Chow variety to all other (possibly singular) projective varieties.

7.4. We can say something similar about Hilbert schemes and state polytopes of [KSZ1]. The Hilbert scheme $\operatorname{Hilb}_{h}\left(\mathbb{P}^{n-1}\right)$ is the set of all subschemes of $\mathrm{P}^{n-1}$ whose Hilbert polynomial is $h$ (fixed). The torus $T=\left(\mathbb{C}^{*}\right)^{n-1}$ acts on $\operatorname{Hilb}_{h}\left(\mathbb{P}^{n-1}\right)$ via a choice of a large enough integer $r>>0$. Let $\mu_{r}$ be the associated moment map. Then as an immediate corollary of $\S 3$ of [KSZ1], we have

7.5. Proposition. Let $I$ be a point in $\operatorname{Hilb}_{h}\left(\mathbb{P}^{n-1}\right)$. The polytope $\mu_{r}(\overline{T \cdot I})$ coincides with the state polytope $\mathrm{St}_{r}(I)$ of $[\mathrm{KSZ} 1]$.

\section{Closing Remarks}

It seems that an algebraic torus action shares very much the properties of the action of a Cartan subgroup. To add more supportive examples, we would like to observe the following.

8.1. Let $k$ be an algebraic closure of the field $\mathbb{F}_{p}$ with $p$ elements. We assume that $X$ is defined over $k$ with an $\mathbb{F}_{p}$-rational structure. Let $F: X \rightarrow X$ be the corresponding Frobenius map. Let $\mathscr{L}_{w}$ denote the intersection cohomology complex (of $l$-adic sheaves, $l$ being a prime different from $p$ ) on $\overline{X_{w}^{a}}, \mathscr{H}^{i}\left(\overline{X_{w}^{a}}\right)$ denote the cohomology sheaf, and $I H^{i}\left(\overline{X_{w}^{a}}\right)$ denote the hypercohomology of $\mathscr{L}_{w}$.

8.2. Following [KL2], we shall say that an irreducible variety $V$ defined over $\mathbb{F}_{p}$ is very pure if for any point $x \in V\left(\mathbb{F}_{p^{r}}\right)$ and for any $i$, all the eigenvalues 
of $\left(F^{*}\right)^{r}$ on $\mathscr{H}_{x}^{i}(V)$ of the cohomology sheaf of the intersection cohomology complex of $V$ have complex absolute value $p^{r i / 2}$.

Since from now on we will only concentrate on the fixed one-parameter subgroup $a \in \mathbf{R}$, we will simply write $X_{w}$ instead of writing $X_{w}^{a}$.

8.3. Theorem. The sheaf $\mathscr{H}^{i}\left(\overline{X_{w}}\right)$ is zero for odd $i$. If $i$ is even and $\overline{X_{w}}$ is defined over the finite field $\mathbb{F}_{p^{r}}$, then all eigenvalues of $\left(F^{r}\right)^{*}$ on $\mathscr{H}_{x}^{i}\left(\overline{X_{w}}\right)$ are equal to $p^{i r / 2}$, where $F$ is the Frobenius map and $x$ is a point on $\bar{X}_{w}$.

Proof. The same as the proof of the corresponding statement in [KL2].

As a consequence of 8.3 , we have the following

8.4. Corollary. The intersection cohomology $I H^{i}\left(\overline{X_{w}}\right)$ of $\overline{X_{w}}$ vanishes in odd degrees. And for even-dimensional intersection cohomology $I H^{i}\left(\overline{X_{w}}\right)$ of $\overline{X_{w}}$, the eigenvalues of the induced Frobenius map are equal to $p^{i / 2}$.

\section{ACKNOWLEDGMENT}

In a recent preprint $[\mathrm{BG}]$, Borovik and Gelfand generalized $(W, Q)$-matroids to Tits systems as well as to a chamber system. I am very grateful to the referee for many very useful suggestions and comments. In particular, $\S 7$, among other things, is suggested by him. I am also indebted to Michel Brion for helping to remove a nonnecessary strong assumption on the Bialynicki-Birula cellular decompositions. It is a great pleasure to thank Robert MacPherson for bringing my attention to this subject and for sending me the preprint [BG]. I should also like to thank A. Borovik for inspiring correspondence.

\section{REFERENCES}

[A] M. F. Atiyah, Convexity and commuting Hamiltonians, Bull. London Math. Soc. 14 (1982), 1-15.

[B-B] A. Bialynicki-Birula, Some theorems on actions of algebraic groups, Ann. of Math. (2) 98 (1973), 480-497.

[BG1] A. Borovik and I. Gelfand, W P-matroids and thin Schubert cells on Tits systems, preprint, 1992.

[BG2] _ Matroids on chamber systems, preprint, 1992.

[CR] H. Crapo and G. Rota, On the foundations of combinatorial theory: combinatorial geometries, M.I.T. Press, Cambridge, MA, 1970.

[D] V. I. Danilov, The geometry of toric varieties, Uspekhi Mat. Nauk. 33 (1978), 85-134; English transl. in Russian Math. Surveys.

[GGMS] I. Gelfand, R. Goresky, R. MacPherson, and V. Serganova, Combinatorial geometries, convex polyhedra, and Schubert cells, Adv. Math. 63 (1987), 301-316.

[GS] I. M. Gelfand and V. V. Serganova, Combinatorial geometries and torus strata on homogeneous compact manifolds, Uspekhi Mat. Nauk 42 (1987), no. 2, 107-134; English transl., Russian Math. Surveys 42 (1987), no. 2, 133-168.

[GuS] V. Guillemin and S. Sternberg, Convexity properties of the moment map, Invent. Math. 67 (1982), 491-513.

[H1] Y. Hu, The geometry and topology of quotient varieties, Ph.D. Thesis, May 1991, M.I.T. The geometry and topology of quotients of torus action (a portion of the above thesis), Duke Math. J. 68 (1992), 151-184.

[H2] _ On the homology of complements of arrangements of subspaces and spheres, Proc. Amer. Math. Soc. 122 (1994), 285-290. 
[KSZ1] M. Kapranov, B. Sturmfels, and A. Zelevinsky, Chow polytopes and general resultants, Duke Math. J. 67 (1992), 198-218.

[KSZ2] _ Quotients of toric varieties, Math. Ann. 290 (1991), 643-655

[KL1] D. Kazhdan and G. Lusztig, Representations of Coxeter groups and Hecke algebras, Invent. Math. 53 (1979), 165-184.

[KL2] Schubert varieties and Poincare duality, Proc. Sympos. Pure Math., vol. 36, Amer. Math. Soc., Providence, RI, 1980, pp. 185-203.

[S] B. Sturmfels, On the matroid stratification of Grassmann varieties, specialization of coordinates, and a problem of N. White, Adv. Math. 75 (1989), 202-211.

Department of Mathematics, University of Michigan, Ann Arbor, Michigan 48109

E-mail address: yihu@math.lsa.umich.edu 